\title{
MAGNETIC TRANSLATIONS FOR A SPATIALLY PERIODIC MAGNETIC FIELD
}

\author{
W. FLOREK* \\ Computational Physics Division, Institute of Physics, A. Mickiewicz University \\ Umultowska 85, 61-614 Poznań, Poland
}

(Received November 29, 1999)

\begin{abstract}
It is shown that in the case of a free electron in a spatially periodic magnetic field the concept of magnetic translations operators is still valid and, moreover, these operators can be defined in the same way as for a Bloch electron in a uniform magnetic field. The results can be a useful tool in the investigation of recently observed phenomena in $2 \mathrm{D}$ electron gas with spatially modulated density.

PACS numbers: 02.20.-a, 73.40.Hm, 71.70.Di, 71.10.-w
\end{abstract}

\section{Introduction}

Recently there has been considerable interest, both experimentally [1] and theoretically $[2,3]$, in the properties of charged particles in different inhomogeneous magnetic field profiles, which has been studied. Since properties of the strongly correlated phases can be obtained from the photoluminescence spectra [2], much effort has been devoted to this technique [4]. This problem is strongly related to the Anderson localization of electrons and, therefore, is important in investigations of high- $T_{\mathrm{c}}$ superconductors and composite-fermions in the quantum Hall effect. Some recent experiments have shown that variation of density in two-dimensional electron systems lead to a fictitious periodic magnetic field [5] and a geometric resonance of the classical cyclotron orbit and the field period. A theory of this effect has been lately proposed by Zimbovskaya and Birman [6].

The aim of this paper is to consider a possibility of introducing magnetic translation operators in the case of a spatially periodic magnetic field and, if this introduction is possible, to determine their form. Some general considerations of two-dimensional quantum systems in a singular vector potential $\boldsymbol{A}$ were presented by Arai [7], who generalized some concepts to nonuniform magnetic fields for continuous quantum systems and presented a method of reduction to lattice quantum systems. A special class of introduced operators $T^{A}$ leave the Hilbert space $L^{2}\left(\mathcal{R}^{2}\right)$

*e-mail: florek@amu.edu.pl 
(or $l^{2}\left(\mathcal{Z}^{2}\right)$ for lattice systems) invariant, so it is possible to construct a continuous version of a Hamiltonian of the Hofstader type [8]. The problem analyzed in the present work is simpler, however it is directly related with the above mentioned phenomena. It is shown that magnetic translation operators introduced independently by Fishbeck [9], Brown [10], and Zak [11] for an electron in a uniform magnetic field and a periodic potential can be also applied in the case of a spatially periodic magnetic field. In the simplest case a free electron is considered.

In the next section a brief summary of the main results for a uniform magnetic field is given, whereas the problem is solved in Sec. 3. Some remarks are presented in the last section.

\section{Bloch electron in homogeneous magnetic field}

- For the sake of simplicity it is assumed that an electron can move in the two-dimensional plane $x y$, whereas the magnetic field $\boldsymbol{H}=H \hat{z}$ is perpendicular to it. Therefore, only the components $A_{x}$ and $A_{y}$ of a vector potential $\boldsymbol{A}$ are relevant. In both cases, i.e. for homogeneous and inhomogeneous magnetic fields, the Hamiltonian is given by the well-known formula

$$
\mathcal{H}=\frac{1}{2 m}\left(p-\frac{e}{c} \boldsymbol{A}\right)^{2}+V(r) .
$$

The only requirement is that $V(r)$ is a periodic function of $r \in \mathcal{R}^{2}$. Positions of crystal nodes are determined by the vectors $\boldsymbol{R}$ of the two-dimensional translation group $\mathcal{T}$ isomorphic to $\mathcal{Z} \times \mathcal{Z}$, so they will be often replaced by pairs $\left(n_{x}, n_{y}\right)$ of integers, i.e. by their coordinates in the crystal base $\left\{a_{1}, a_{2}\right\}$. Since a point group symmetry is not taken into account, the square lattice with $a_{1} \perp a_{2}$ and $a_{1}=a_{2}=a$ is considered.

\subsection{A form of the vector potential}

For a constant and uniform magnetic field the vector potential $\boldsymbol{A}$ can be written as linear function of the position vector $r$ [12]. Moreover, the coordinates $A_{x}$ and $A_{y}$ can be chosen in such a way that they do not depend on $x$ and $y$, respectively ${ }^{\dagger}$. Therefore in the most general case one obtains

$$
A_{x}=\alpha y \text { and } A_{y}=\beta x .
$$

The real numbers $\alpha, \beta$ have to satisfy

$$
H=\beta-\alpha
$$

for a given magnitude $H$ of the magnetic field. Of course, this form includes the antisymmetric gauge $(r \times \boldsymbol{H}) / 2$ for $\alpha=-\beta=-H / 2$ and the Landau gauge for $\beta=H, \alpha=0$.

\footnotetext{
†This condition is stronger than the radiation gauge $\nabla \cdot \boldsymbol{A}=0$.
} 


\subsection{The symmetry of a problem}

The periodicity of $V(r)$ yields the two-dimensional translation group $\mathcal{T} \simeq$ $\mathcal{Z} \times \mathcal{Z}$ as the symmetry group of the Hamiltonian. Therefore, one can introduce concepts of quantized quasi-momentum, energy bands and Bloch electrons. Irreducible representations of $\mathcal{T}$ label energy levels of $\mathcal{H}$. However, in the presence of the magnetic field $H \hat{z}$, at least except for special values of $H$, projective representations have to be used and their factor systems depend on the magnetic flux through the unit crystal cell. The periodic boundary conditions imposed on (projective) representation $T(\boldsymbol{R})$ leads to magnetic flux quantization and the concept of magnetic cells (or magnetic periodicity) $[10,12]$. The periodic boundary conditions are also responsible for choosing the magnetic field perpendicular to a crystal plane $[10,11,13]$. It should be underlined that in Zak's approach projective representations of $\mathcal{T}$ are replaced by vector representations of a central extension of $\mathcal{T}$ by a group of factors included in U(1). Of course, these two approaches are equivalent.

\subsection{Projective representations}

Operators of a projective irreducible representation of $\mathcal{T}$ which commute with $\mathcal{H}$ can be chosen as $[9-12,14]$

$$
T(\boldsymbol{R})=\exp \left[\boldsymbol{R} \cdot\left(p-\frac{e}{c} \boldsymbol{A}^{\prime}\right)\right]
$$

where $\boldsymbol{A}^{\prime}$ is a vector potential associated with $\boldsymbol{A}$ and, in these simplified considerations, given as

$$
A_{x}^{\prime}=\beta y \text { and } A_{y}^{\prime}=\alpha x .
$$

The commutation $T(\boldsymbol{R})$ with $\mathcal{H}$ follows from the fact that the coordinates of $\boldsymbol{\pi}=\boldsymbol{p}-\frac{e}{c} \boldsymbol{A}$ commute with the coordinates of $\boldsymbol{\pi}^{\prime}=p-\frac{e}{c} \boldsymbol{A}^{\prime}$ [10-12]. Moreover, the commutators $\left[\pi_{x}, \pi_{y}\right],\left[\pi_{x}^{\prime}, \pi_{x}^{\prime}\right]$ etc. are numbers, so they commute with any other operator. This fact is very important in the derivation of a factor system for the representation $T(\boldsymbol{R})$. This factor system and the group-theoretical commutator $T(\boldsymbol{R}) T\left(\boldsymbol{R}^{\prime}\right) T(\boldsymbol{R})^{-1} T\left(\boldsymbol{R}^{\prime}\right)^{-1}$ depend only on the magnitude $H$, not on the form of the vector potential. However, working with a local gauge $\boldsymbol{A}_{\boldsymbol{R}}^{\prime}$ one can change a factor system, but the commutator is unaffected (is gauge-independent) [15].

\subsection{Movement of a Bloch electron}

In the case of homogeneous magnetic field an electron moves around the cyclotron orbit with coordinates of the center given by operators $\pi_{y}^{\prime}, \pi_{x}^{\prime}$. This movement is quantized and is related to the broadening of the Landau levels [10,16].

\section{Inhomogeneous magnetic field}

In order to keep the symmetry described as the translation group one has to assume that the non-constant terms in the magnetic field magnitude are periodic with respect to $x$ and $y$ coordinates. Therefore, $\pi^{2}$, the generalized kinetic term of the Hamiltonian (1), is invariant under $\boldsymbol{R} \in \mathcal{T}$ and, at least in the first-order 
approximation, the potential $V(r)$ can be omitted. Applying the Fourier transform, a periodic magnetic field can be written as a sum of the sine and cosine functions $\sin k_{x} x, \sin k_{y} y$ (or $\exp (\mathrm{i} k \cdot r)$, if one prefers the complex analysis), so, to simplify the problem, it is assumed that

$$
H=H_{0}+H_{1}[\cos (k x)+\cos (k y)],
$$

where $k=k_{x}=k_{y}=2 \pi / a$.

\subsection{The vector potential}

For the magnetic field given by (5) the vector potential $\boldsymbol{A}$ can be chosen in many gauge invariant forms. For the sake of clarity the simplest form in the radiation gauge $(\nabla \cdot A=0)$ is assumed

$$
\begin{aligned}
& A_{x}=\alpha y-\frac{H_{1}}{k} \sin (k y), \\
& A_{y}=\beta x+\frac{H_{1}}{k} \sin (k x),
\end{aligned}
$$

with $\beta-\alpha=H_{0}$.

\subsection{The symmetry group}

Due to the periodicity of $H$ the symmetry group is still $\mathcal{T} \simeq \mathcal{Z} \times \mathcal{Z}$. Its irreducible representations should label eigenspaces of $\mathcal{H}$. There is, however, one problem: "Does it suffice to consider projective representations or, due to higher terms of $x$ and $y$ in $A_{x}, A_{y}$, and $H$, some more complex structure should be used?" Within the frame of Zak's approach it may mean that one has to investigate non-Abelian extensions, for example. It seems that when for $\boldsymbol{A}$ being linear function of $r$ the second cohomology group (related with projective representations or, equivalently, with central extensions) comes into play, then for $A_{x}, A_{y}$ being second order functions of $x, y$ the third cohomology group should be considered etc. There are some hints from mathematics and physics which indicate that it suffices to limit consideration to projective representations (i.e. the second cohomology group).

At first, we are interested in $\boldsymbol{A}$ and $H$ expressed by the (co-)sine or other periodic functions with an infinite Taylor expansion, so - if the order of a cohomology group depends on the order of functions - the cohomology group of the infinite order should be taken into account. Moreover, factor systems of projective representations in the case of a homogeneous magnetic field depend on the magnetic flux through the unit crystal cell. This quantity is always a number calculated as an integral over the unit cell of the product $H(x, y) \mathrm{d} x \mathrm{~d} y$ (or as an integral over the edges of a cell $\left.\int \boldsymbol{A}(\boldsymbol{r}) \cdot \mathrm{d} \boldsymbol{r}[7]\right)$. Such an integral depends only on the constant term $H_{0}$ since the integration of periodic terms ${ }^{\ddagger}$ gives zero. The cohomology group of order $n$ demands considerations of the $n$-th co-boundaries and co-cycles, which involve $n$ lattice vectors. It seems it would be necessary in the case of a hypothetical "field" being a tensor of rank $n\left(F_{x y z}, G_{x y z t}\right.$, etc. $)$ - in the case of

\footnotetext{
$\ddagger$ In this place a "periodic term" denotes all but a constant term of the Fourier transform.
} 
magnetic field, which can be described by the tensor $H_{x y}$, the most important are loops (drawn in two dimensions) encircling the magnetic flux (a one-dimensional object). At last, there are no well-investigated group-theoretical generalizations of non-Abelian extensions corresponding to fourth, fifth etc. cohomology groups (at least the author is not aware of such considerations).

\subsection{Projective representations}

In this section a form projective representation $T$ of $\mathcal{T}$ is derived. This representation should commute with $\mathcal{H}$ and the results for the uniform magnetic field have to be revealed in the limit $H_{1} \rightarrow 0$.

Let us assume that operators of a projective representation have the following form:

$$
T(\boldsymbol{R})=\exp \left(-\frac{\mathrm{i}}{\hbar} \boldsymbol{\pi}^{\prime} \cdot \boldsymbol{R}\right),
$$

where a form of the operator $\boldsymbol{\pi}^{\prime}$ depends on $\boldsymbol{A}$ and in the limit $H_{1} \rightarrow 0$ we have $\boldsymbol{\pi}^{\prime}=p-(e / c) \boldsymbol{A}^{\prime}$ with $\boldsymbol{A}^{\prime}$ defined in the previous section by Eq. (4). Due to the periodicity of $V(r)$ (moreover, $V(r)=0$ is assumed in the simplest approximation) it is enough to calculate a commutator $\left[T(\boldsymbol{R}), \boldsymbol{\pi}^{2}\right]$. If $\pi_{x}$ and $\pi_{y}$ commute with $T(\boldsymbol{R})$ then also $\mathcal{H}$ does. The condition $\left[T(\boldsymbol{R}), \pi_{x}\right]=\left[T(\boldsymbol{R}), \pi_{y}\right]=0$ allows also labeling of eigenspaces of the canonical momenta $\pi_{x}$ and $\pi_{y}$ by irreducible projective representations of $\mathcal{T}$. Substituting $P=-\frac{\mathrm{i}}{\hbar} \boldsymbol{\pi}^{\prime} \cdot \boldsymbol{R}$ one can write

$$
T(\boldsymbol{R})=\exp P=\sum_{n=0}^{\infty} \frac{P^{n}}{n !}
$$

and

$$
\left[\exp P, \pi_{\xi}\right]=\sum_{n=0}^{\infty} \frac{1}{n !}\left[P^{n}, \pi_{\xi}\right], \quad \xi=x, y .
$$

The last commutator equals

$$
\left[P^{n}, \pi_{\xi}\right]=\sum_{k=0}^{n-1}\left(\begin{array}{l}
n \\
k
\end{array}\right) C_{n-k}^{\xi} P^{k}
$$

where

$$
C_{l}^{\xi}=\left[P, C_{l-1}^{\xi}\right] \text { and } C_{0}^{\xi}=\pi_{\xi} .
$$

These formulae yield that $C_{1}^{\xi}=\left[P, \pi_{\xi}\right]=0$ is a sufficient condition for $[T(\boldsymbol{R}), \mathcal{H}]=0$. For example, it can be solved in the case of the homogeneous magnetic field, i.e. when $\pi$ is a linear function of $x$ and $y$, and this solution was found by Brown and Zak and then generalized to any linear vector potential by the author (see also [9]). However, the case of the inhomogeneous magnetic field yields $\boldsymbol{A}$ being a square (or higher order) function of coordinates $x, y$ and the condition $C_{1}^{x}=0$ does not lead to any non-trivial solutions for $P$. Therefore, the formula (9) has to be substituted to the condition (8) and the solution has to be found in this more general case. Therefore, one obtains

$$
\left[\exp P, \pi_{\xi}\right]=\sum_{n=0}^{\infty} \frac{1}{n !} \sum_{k=0}^{n-1}\left(\begin{array}{l}
n \\
k
\end{array}\right) C_{n-k}^{\xi} P^{k}=\left(\sum_{n=1}^{\infty} \frac{1}{n !} C_{n}^{\xi}\right) \exp P .
$$


Therefore, the sum of commutators $C_{n}^{\xi}$ has to be equal to zero, which, is satisfied by the special solution $C_{1}^{\xi}=0$. After the substitution $\pi_{\xi}^{\prime}=-\mathrm{i} \hbar \partial_{\xi}-(e / c) A_{\xi}^{\prime}$ the operator $P=-\frac{i}{\hbar} X \pi_{x}^{\prime}-\frac{i}{\hbar} Y \pi_{y}^{\prime}$, where $X=n_{x} a, Y=n_{y} a$ are coordinates of $\boldsymbol{R}$, decomposes into two summands

$$
P=P_{1}+P_{2} ; \quad P_{1}=-\left(X \partial_{x}+Y \partial_{y}\right), \quad P_{2}=\frac{\mathrm{i} e}{\hbar c}\left(X A_{x}^{\prime}+Y A_{y}^{\prime}\right) .
$$

Therefore, in a general case, we have

$$
C_{1}^{\xi}=\left[P, \pi_{\xi}\right]=\frac{e}{c}\left[\left(X \partial_{x}+Y \partial_{y}\right) A_{\xi}-\partial_{\xi}\left(X A_{x}^{\prime}+Y A_{y}^{\prime}\right)\right] .
$$

If one assumes that $\partial_{x} A_{x}=\partial_{y} A_{y}=0$, this can be simplified to

$$
\begin{aligned}
C_{1}^{x} & =\frac{e}{c}\left[Y \partial_{y} A_{x}-\partial_{x}\left(X A_{x}^{\prime}+Y A_{y}^{\prime}\right)\right], \\
C_{1}^{y} & =\frac{e}{c}\left[X \partial_{x} A_{y}-\partial_{y}\left(X A_{x}^{\prime}+Y A_{y}^{\prime}\right)\right] .
\end{aligned}
$$

Example. Let us consider the case $A_{x}=\alpha y$ and $A_{y}=\beta x+H_{1} x^{2} / 2$. We are looking for a solution in a similar form, i.e. determined by an associated vector potential $\boldsymbol{A}^{\prime}$ being a square function of $x$ and $y$, so we assume that

$$
A_{x}^{\prime}=\alpha^{\prime} y+\alpha^{\prime \prime} y^{2} \text { and } A_{y}^{\prime}=\beta^{\prime} x+\beta^{\prime \prime} x^{2} .
$$

Equation (13) gives

$$
C_{1}^{x}=\frac{e}{c} Y\left(\alpha-\beta^{\prime}-2 \beta^{\prime \prime} x\right), \quad C_{1}^{y}=\frac{e}{c} X\left(\beta+H_{1} x-\alpha^{\prime}-2 \alpha^{\prime \prime} y\right) .
$$

Since $C_{1}^{\xi} \neq 0$ in a general case, we have to calculate $C_{2}^{\xi}$ (note that only $P_{1}$ is relevant):

$$
C_{2}^{x}=\left[P, C_{1}^{x}\right]=2 \beta^{\prime \prime} \frac{e}{c} X Y, \quad C_{2}^{y}=\left[P, C_{1}^{y}\right]=\frac{e}{c} X\left(-X H_{1}+2 Y \alpha^{\prime \prime}\right) .
$$

Therefore $C_{l}^{\xi}=0$ for $l \geq 3$ and the commutator $\left[\exp P, \pi_{\xi}\right]=0$ if $C_{1}^{\xi}+C_{2}^{\xi} / 2=0$ for any $x, y, X, Y$. For $\xi=x$ we obtain

$$
\beta^{\prime \prime}=0 \text { and } \beta^{\prime}=\alpha \text {. }
$$

It is easy to notice that a solution for $\xi=y$ and $H_{1} \neq 0$ can be obtained only when $\alpha^{\prime \prime}=0$ and $X=0$. This means that the (magnetic) translations $T([0, Y]$ ) commute with the Hamiltonian. This is not surprising since the magnetic field $H=(\beta-\alpha)+H_{1} x$ is not periodic in the $x$-th direction.

This example shows that the linear term in $A_{\xi}$ (the constant term in $H$ ) always appears in $A_{\xi}^{\prime}$ in the same way as in the case of the homogeneous magnetic field. Therefore, for the vector potential given by Eq. (6) we choose the associated potential in the following form:

$$
A_{x}^{\prime}=\beta y+f(x, y), \quad A_{y}^{\prime}=\alpha x+g(x, y),
$$

where the functions $f$ and $g$ will be determined from the commutation conditions. Substituting into Eq. (13) we obtain

$$
\begin{aligned}
& C_{1}^{x}=-\frac{e}{c}\left\{Y H_{1} \cos (k y)+\partial_{x}[X f(x, y)+Y g(x, y)]\right\}, \\
& C_{1}^{y}=\frac{e}{c}\left\{X H_{1} \cos (k x)-\partial_{y}[X f(x, y)+Y g(x, y)]\right\} .
\end{aligned}
$$


Next the commutators will be determined with the use of the operator $P_{1}$, see Eq. (11), and derivatives of each function will be considered separately. The first part of $C_{1}^{x}$, up to the constant factor $-H_{1} e / k c$, gives the following series:

$$
\begin{gathered}
k Y \cos (k y),(k Y)^{2} \sin (k y),-(k Y)^{3} \cos (k y), \\
-(k Y)^{4} \sin (k y),(k Y)^{5} \cos (k y), \ldots
\end{gathered}
$$

Decomposing it into two series with $\cos (k y)$ and $\sin (k y)$, respectively, and substituting it into the infinite sum in Eq. (10) one obtains (taking into account that $k=2 \pi / a$ and $Y=n_{y} a$, so $\left.k Y=2 n_{y} \pi\right)$

$$
\begin{aligned}
& \cos (k y) \sum_{n=0}^{\infty} \frac{(-1)^{n}(k Y)^{2 n+1}}{(2 n+1) !}+\sin (k y)\left[1-\sum_{n=0}^{\infty} \frac{(-1)^{n}(k Y)^{2 n}}{(2 n) !}\right] \\
& =\cos (k y) \sin (k Y)+\sin (k y)[1-\cos (k Y)]=0 .
\end{aligned}
$$

The same result is obtained for the part of $C_{1}^{y}$ containing $\cos (k x)$. Therefore, the operators $T(\boldsymbol{R})$ commute with the Hamiltonian for the trivial functions

$$
f(x, y) \equiv g(x, y) \equiv 0 \text {. }
$$

This is not so surprising if we recall that the factor system depends on the magnetic flux through a lattice cell [15] and the periodic part of $H$ gives no input to it. Such a solution leads to an interesting difference between $H$ and $H^{\prime}$ : the first is periodic, whereas the second is uniform since $H^{\prime}=-H_{0}$. This difference is more evident if we take into account the vector potentials $\boldsymbol{A}$ and $\boldsymbol{A}^{\prime}$ : the original one satisfies the inhomogeneous wave equation [17]:

$$
\nabla^{2} \boldsymbol{A}=-\frac{4 \pi}{c} \boldsymbol{J}=k H_{1}[\sin (k y),-\sin (k x)],
$$

whereas $\nabla^{2} A^{\prime}=0$.

The obtained solution implies that a spatially periodic magnetic field does not require any changes in the group of magnetic translations (projective representations of the translation group).

\subsection{Other solutions}

It is interesting to check what happens in the case when $A_{\xi}^{\prime}$ depends on $x$ and $y$ in a similar way as $A_{\xi}$. To begin with we choose

$$
f(y)=\frac{H_{1}}{k} \sin (k y), \quad g(x)=-\frac{H_{1}}{k} \sin (k x) .
$$

Such a choice leads to an associated magnetic field $H^{\prime}$ equal to

$$
H^{\prime}=(\alpha-\beta)-H_{1}[\cos (k x)+\cos (k y)]=-H,
$$

as we have obtained in the case of the homogeneous magnetic field (cf. [15]). The series of commutators $C_{l}^{x}$ containing $g(x)$ and its derivatives is calculated as follows (up to the constant factor $H_{1} Y e / k X c$ ) :

$$
\begin{aligned}
& k X \cos (k x),(k X)^{2} \sin (k x),-(k X)^{3} \cos (k x), \\
& -(k X)^{4} \sin (k x),(k X)^{5} \cos (k x), \ldots
\end{aligned}
$$


Substituting into the infinite sum in Eq. (10) one obtains, as in Eq. (16),

$$
\cos (k y) \sin (k Y)+\sin (k y)[1-\cos (k Y)]=0 \text {. }
$$

However, this solution has been obtained after division by $X$, so it is valid only for $X \neq 0$. Analogous considerations for $f(y)$ lead to the same result, but now $Y \neq 0$. Therefore, the potential obtained for functions (17) determines the operators commuting with the Hamiltonian only for $X, Y \neq 0$ or $X=Y=0$. Exclusion of the axis $X=0$ is caused by the lack of one power of $X$. It can be revealed if the magnetic translations are defined locally [15], i.e. the form of functions $f$ and $g$ will depend on $X$ and $Y$. The simplest solution is to put

$$
f_{Y}(y)=Y f(y) \text { and } g_{X}(x)=X g(x) .
$$

Another way is realized by switching $f$ and $g$, so

$$
f(x)=\frac{H_{1}}{k} \sin (k x), \quad g(y)=-\frac{H_{1}}{k} \sin (k y) .
$$

In this case, however, $H^{\prime}=-H_{0}$ (without the periodic term) and, moreover the vector potential $\boldsymbol{A}^{\prime}$ is no longer written in the radiation gauge.

\subsection{Movement of a Bloch electron}

The approximated considerations of an electron in spatially inhomogeneous field presented in many textbooks, e.g. [17], show that for small values of $\nabla H$ a charged particle gains an additional velocity perpendicular to $\boldsymbol{H}$ and $\nabla H$. Since $\nabla H$ is perpendicular to lines on which $\boldsymbol{H}$ is constant then it is most likely that the orbit center will move along these lines. Results of simple numerical simulations (only the Lorentz force for a periodic magnetic field has been taken into account) seem to confirm this picture. However, a system is very unstable and actual behavior strongly dependson the starting values and accuracy. When the time-step

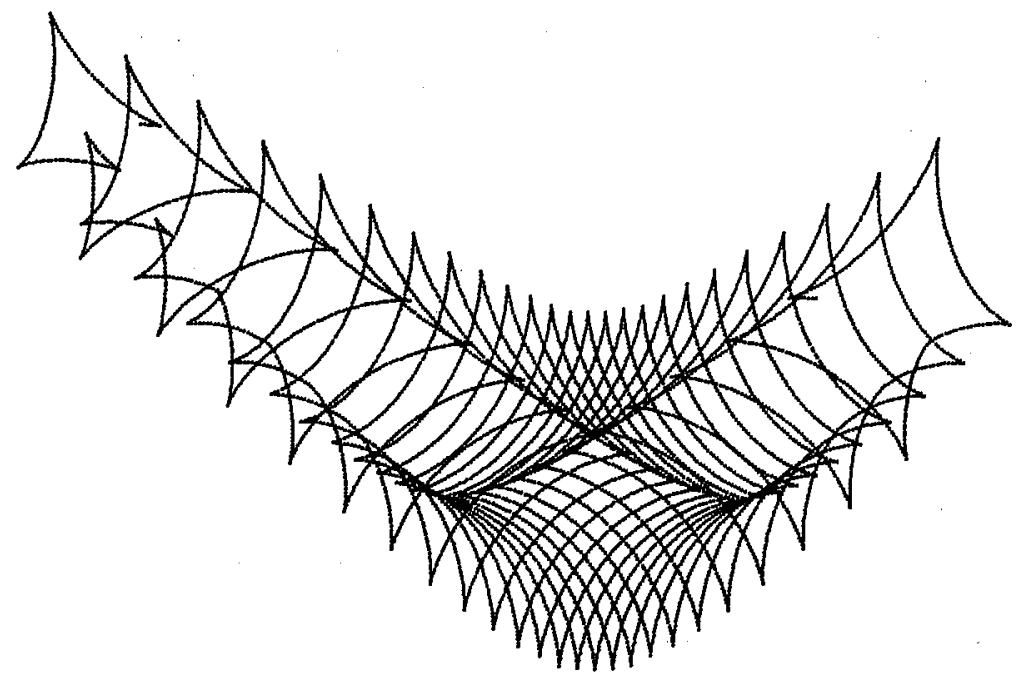

Fig. 1. A trajectory of the orbit center for a large orbit radius $\rho \gg a$. 
has been relatively large $(\omega \Delta t \approx 0.01)$, the center moved towards the maximum (for large $H$ ) or minimum (for small $H$ ) of the function $H(x, y)$. This movement was almost negligible for small values of $\Delta t(\omega \Delta t \approx 0.0001)$. A critical value of $H$ depends on the particle velocity and corresponds to the orbit diameter $2 \rho$ close to the field period, cf. [5]. It could be expected that in the limit case $\Delta t \rightarrow \mathrm{d} t$ the orbit center would be "stabilized" on an "equipotential" line $H=$ const. The other conclusions are almost obvious: (i) in a strong magnetic field (a very small orbit diameter) the movement of the orbit center is much slower since the particle moves in almost constant magnetic field; (ii) in the case of small magnetic field (a large orbit diameter) the particle moves in regions of different magnetic field magnitudes and the orbit center trajectory is not a smooth line (see Fig. 1).

\section{Final remarks}

It has been shown that a spatially periodic magnetic field leads to the same magnetic translation operators as in the case of a uniform field and a periodic potential $V(r)$. Since the results obtained do not depend on an actual value of $k$, then they are valid for any periodic function of $x$ and $y$ written as its Fourier transform. When a periodic potential $V(r)$ is also present then our considerations are applicable to the case of commensurate periods of $H$ and $V$ only. The results of numerical simulations suggest that the orbit center moves along an "equipotential" line $H=$ const. This movement is more stable in the case of relatively large fields, i.e. for the orbit radius $\rho$ smaller than the field period $a$. The magnetic translation operators cannot be interpreted as the position of the orbit center [15, 18], since the latter is not a constant of motion. They rather correspond to the center of the orbit center trajectory. It should be underlined that this work does not present a theory of the observed effects [5], but provides a mathematical tool, which can be useful in such investigations.

\section{Acknowledgments}

This work is supported by the Committee for Scientific Research within the project No. 263/P03/99/16.

\section{References}

[1] See, e.g., W. Petrich, M.H. Anderson, J.R. Ensher, E.A. Cornell, Phys. Rev. Lett. 74, 3352 (1995); A.K. Geim, S.V. Dubonos, J.G.S. Lok, I.V. Grigorieva, J.C. Maan, L. Theil Hansen, P.E. Lindelof, Appl. Phys. Lett. 71, 2379 (1997); A.K. Geim, I.V. Grigorieva, S.V. Dubonos, J.G.S. Lok, J.C. Maan, P.E. Lindelof, F.M. Peeters, Nature 390, 259 (1997); P.C.M. Christianen, F. Piazza, J.G.S. Lok, J.C. Maan, W. van der Vleuten, Physica B 249-251, 624 (1998); V. Kubrak, F. Rahman, B.L. Gallagher, P.C. Main, M. Henini, C.H. Marrows, M.A. Howson, Appl. Phys. Lett. 74, 2507 (1999); see also F.M. Peeters, J. De Boeck, in: Handbook of Nanostructured Materials and Nanotechnology, Ed. H.S. Nalwa, Vol. 3, Academic Press, New York 1999, p. 345 for a recent review.

[2] N.R. Cooper, Phys. Rev. B 53, 10804 (1996); N.R. Cooper, D.B. Chklovskii, Phys. Rev. $B$ 55, 2436 (1997). 
[3] A.B. Dzyubenko, JETP Lett. 66, 617 (1997); D.M. Whittaker, A.J. Shields, Phys. Rev. B 56, 15185 (1997); J. Reijniers, F.M. Peters, A. Matulis, Phys. Rev. B 59, 2817 (1999); J.A.K. Freire, A. Matulis, F.M. Peters, V.N. Freire, G.A. Farias, Phys. Rev. B 61, 2895 (2000); H. Potempa, L. Schweitzer, Ann. Phys. (Leipzig) 8, Special Issue, SI-209 (1999); A.B. Dzyubenko, A.Yu. Sivachenko, submitted to Physica E, preprint available on http://xxx.lanl.gov/cond-mat/9908406.

[4] See, for example, W. Ossau, D.R. Yakovlev, U. Zehnder, G.V. Astakhov, A.V. Platonov, V.P. Kochereshko, J. Nürnberger, W. Faschinger, M. Keim, A. Waag, G. Landwehr, P.C.M. Christianen, J.C. Maan, N.A. Gippius, S.G. Tikhodeev, Physica $B$ 256-258, 323 (1998); C. Penn, P.C.M. Christianen, F. Schäffler, J.C. Mann, G. Bauer, Physica B 256-258, 363 (1998); M. Hayne, C.L. Jones, R. Bogaerts, C. Riva, A. Usher, F.M. Peters, F. Herlach, V.V. Moshchalkov, M. Henini, Phys. Rev. B 59, 2927 (1999) and references quoted therein.

[5] S.D.M. Zwerschke, R.R. Gerhardts, Phys. Rev. Lett. 83, 2616 (1999); J.H. Smet,

- S. Jobst, K. von Klitzing, D. Weiss, W. Wegscheider, V. Umansky, ibid., 2620; R.L. Willett, K.W. West, L.N. Pfeiffer, ibid., 2624.

[6] N.A. Zimbovskaya, J.L. Birman, Phys. Rev. B 60, 16762 (1999); submitted to Phys. Rev. B, preprint available on http://xxx.lanl.gov/cond-mat/9908184.

[7] A. Arai, J. Math. Phys. 39, 2476 (1998).

[8] Ch. Kreft, R. Seiler, J. Math. Phys. 37, 5207 (1996).

[9] H.J. Fishbeck, Phys. Status Solidi 3, 1082 (1963).

[10] E. Brown, Phys. Rev. 133, A1038 (1964).

[11] J. Zak, Phys. Rev. 134, A1602, A1607 (1964).

[12] W. Florek, S. Wałcerz, J. Math. Phys. 39, 739 (1998).

[13] W. Florek, Rep. Math. Phys. 38, 235 (1996); W. Florek, M. Thomas, Proc. European Conference "Physics of Magnetism '99", Poznan 1999, Acta Phys. Pol. A, in press.

[14] B. Huckestein, Rev. Mod. Phys, 67, 357 (1995).

[15] W. Florek, Acta Phys. Pol. A 92, 399 (1997).

[16] J. Zak, Phys. Rev. 136, A776 (1964).

[17] J.D. Jackson, Classical Electrodynamics, Wiley, New York 1962, Ch. 6.5.

[18] M.M. Johnson, B.A. Lipmann, Phys. Rev. 76, 829 (1949). 